\title{
Energy Release in Driven Twisted Coronal Loops
}

\author{
M.R. Bareford ${ }^{1}$ • M. Gordovskyy ${ }^{2}$. \\ P.K. Browning ${ }^{2}$ - A.W. Hood ${ }^{1}$
}

(C) Springer $\bullet \bullet \bullet \bullet$

\begin{abstract}
Magnetic reconnection in twisted magnetic fluxtubes, representing coronal loops, is investigated. The main goal is to establish the influence of the field geometry and various thermodynamic effects on the stability of twisted fluxtubes and on the size and distribution of heated regions. In particular, we aim to investigate to what extent the earlier idealised models, based on the initially cylindrically symmetric fluxtubes, are different from more realistic models, including the large-scale curvature, atmospheric stratification, thermal conduction and other effects. In addition, we compare the roles of Ohmic heating and shock heating in energy conversion during magnetic reconnection in twisted loops. The models with straight fluxtubes show similar distribution of heated plasma during the reconnection: it initially forms a helical shape, which subsequently becomes very fragmented. The heating in these models is rather unformly distributed along fluxtubes. At the same time, the hot plasma regions in curved loops are asymmetric, and consentrate close to the loop tops. Large-scale curvature has a destabiling influence: lower twist is needed for instability. Footpoint convergence normally delays instability slightly, although, in some cases converging fluxtubes can be less stable. Finally, introducing a stratified atmosphere gives rise to decaying wave propagation, which has destabilising effect.
\end{abstract}

Keywords: Instabilities; Magnetic fields; Magnetohydrodynamics; Corona

\section{Introduction}

The solar corona is thought to be heated to temperatures of millions of degrees Kelvin by dissipation of stored magnetic energy. The details of the processes of energy storage and dissipation remain contentious, and it is likely that the corona is heated by a combination of mechanisms (Parnell and De Moortel, 2012). A very plausible scenario, especially for heating in active regions, is that the corona is heated by the combined effect of many small flare-like events known

\footnotetext{
1 School of Mathematics and Statistics, University of St Andrews, St Andrews, Fife KY16 9SS, UK email: awh@st-andrews.ac.uk

${ }^{2}$ School of Physics and Astronomy, University of Manchester, Manchester M13 9PL, UK
} 
as "nanoflares" (Parker, 1988). Thus, understanding of flares, especially smaller events, contributes to solving the coronal heating problem. It is essential to understand the heating of loops, which are the main building blocks of the coronal magnetic field (Reale, 2014).

Twisted magnetic fields are likely to be very common in the solar corona, and twist is associated with free magnetic energy. Indeed, all non-potential loops need to be twisted to some extent to remain in equilibrium in a high- $\beta$ plasma. Furthermore, new magnetic flux ropes emerging from below the solar surface should already be twisted, and further twisting is produced by photospheric footpoint motions with vorticity. In essence, the magnetic fields that permeate the solar corona acquire free energy due to the convective motions that take place in and around the loop footpoints: i.e., where the field intersects the photosphere. There is an increasing body of observational evidence for solar flares occurring in twisted coronal loops, some of which are directly interpreted as kink instabilities (see Srivastava et al., 2010; Kuridze et al., 2013; Kumar and Cho, 2014; Yan et al., 2014; Wang et al., 2015). In addition, high resolution observations from HiC show untwisting of "braided" fields associated with energy release (Cirtain et al., 2013), which appear, in fact, to match the kink-unstable twisted loops described by Cargill (2013).

Here, we focus on the energy release within a single twisted magnetic fluxtube, representing a coronal loop. The primary emphasis is on modelling microflares and similar events, but the combined effect of many such events with different magnitudes also provides an effective coronal heating mechanism (Browning and Van der Linden, 2003; Bareford et al., 2010, 2011).

Previously, numerical simulations have shown that magnetic energy release, sufficient for coronal heating above active regions, can occur as a consequence of an ideal instability (Browning et al., 2008; Hood et al., 2009; Botha et al., 2011; Bareford et al., 2013). As the free magnetic energy increases with the twist angle, the helical coronal loops become more and more susceptible to the kink instability (Hood, 1992). This instability, although in itself an ideal process, triggers the formation of multiple current sheets and leads to magnetic reconnection at many sites within the loop volume. Magnetic reconnection causes energy to be released from the field, heating the coronal plasma.

Previous models of this process start with a field configuration that is known to be already unstable (i.e. slightly beyond the threshold for linear ideal kink instability). In addition, the field is usually modelled as a simple straight cylinder where the field strength depends on the distance from the loop axis only. In reality, coronal magnetic fields must expand from localised photospheric sources - despite the much-discussed phenomenon of "constant cross-section", which now seems more likely to reflect the plasma emission (Klimchuk, 2000; Peter and Bingert, 2012). Furthermore, coronal loops are also inevitably curved.

These simplifications may affect the dynamics and energetics of twisted loops, artificially restricting or exaggerating the amount of magnetic energy released. As well as the field geometry, features involving atmospheric physics, which are expected to influence how well magnetic energy is thermalised within the loop volume, are also usually ignored. For example, most previous work uses a 
simplified adiabatic energy equation, although the effects of thermal conduction in cylindrical unstable loops have been considered by Botha et al. (2011).

Recently, Gordovskyy et al. (2014) investigated the kink instability and magnetic reconnection in more realistic configurations with large-scale curvature (i.e. loop-like fluxtubes) and atmospheric stratification. Although the key focus of that study is particle acceleration, it has revealed some features not present in idealised cylindrical models, such as the loop contraction, asymmetric current distribution and variation of the energy release along the loop. Hence, the loop topology and various thermodynamic effects can substantially affect the energy release process and, therefore, they need to be investigated in more detail.

The aim of this paper is to consider heating in twisted coronal loops, involving more realistic models, and, along the way, we explore the effects of removing various idealisations and simplifications which have been adopted in previous works. Does the basic mechanism of an ideal kink instability triggering the formation of fragmented current sheets, leading to significant dissipation of magnetic energy, persist in a realistic loop model? If so, how are the dynamics and energetics affected by the magnetic field geometry and the plasma physics incorporated within the modelling? Therefore, we investigate a family of five loop models which allow us to compare and contrast the effects of field geometry, both loop curvature and field expansion/convergence. In contrast to previous models which use analytically-derived kink-unstable fluxtubes, we use kink-unstable configurations obtained by rotating foot-points of initially potential fluxtubes/loops and, hence, have much greater flexibility in choice of loop configurations. Furthermore, our models are made more realistic by incorporating thermal conduction. Finally, one of the models has an atmosphere stratified in both density and temperature, thus representing a loop whose lower layers are embedded in the upper chromosphere.

The paper is structured in the following manner. Section 2 describes the different loop configurations used for the nonlinear magnetohydrodynamic (MHD) simulations. Section 3 presents the numerical code and Section 4 focuses on how results change as the loop model is made more and more realistic: e.g., how the energy release is affected by the addition of curvature, or how the results change when a stratified atmosphere is used. Finally, in the last section, the results are summarised and our conclusions are given.

\section{Loop Configurations}

The primary goal of this study is to investigate the effect of field geometry on the energy release in twisted loops. Hence, our simulations cover four separate field configurations, see Figure 1. In models A and B, we consider straight fluxtubes using a domain with dimensions $x= \pm 5 \mathrm{Mm}, y= \pm 5 \mathrm{Mm}, z= \pm 10 \mathrm{Mm}$, with the fluxtubes initially orientated along the z-axis. The first fluxtube (A) has a constant cross section, whereas model $\mathrm{B}$ has a field converging towards the footpoints: the field strength at the footpoints is twice the value at the centre (or apex) of the fluxtube. The purpose of loop B then is to show the influence of field convergence with respect to the kink instability and subsequent heating. 

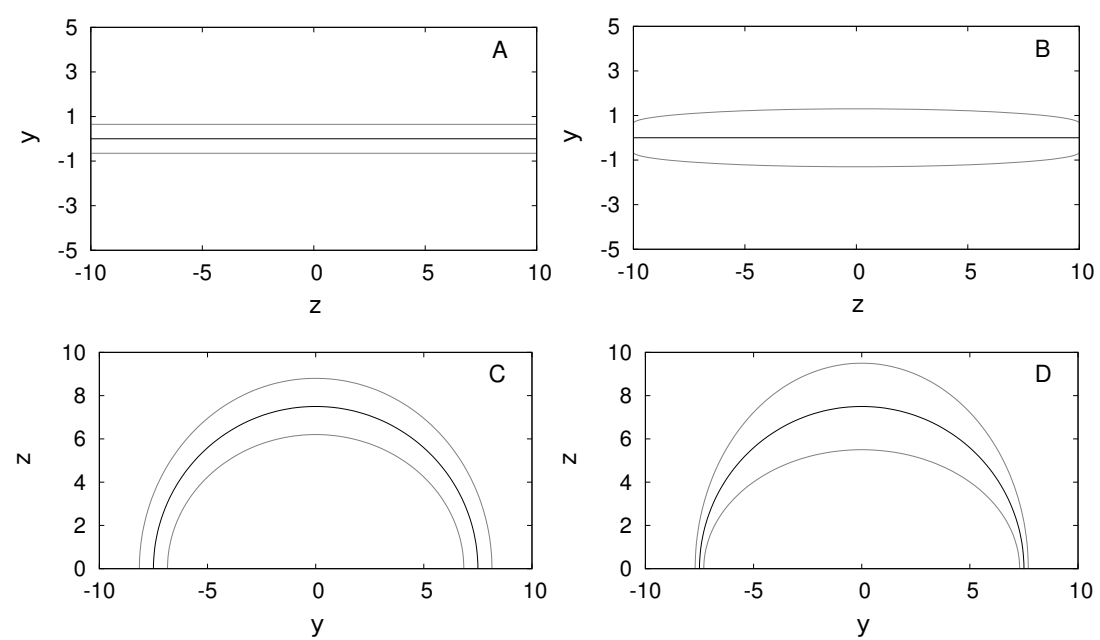

Figure 1. Initial magnetic field geometry for loops A (top left), B (top right), C (bottom left), and D (bottom right).
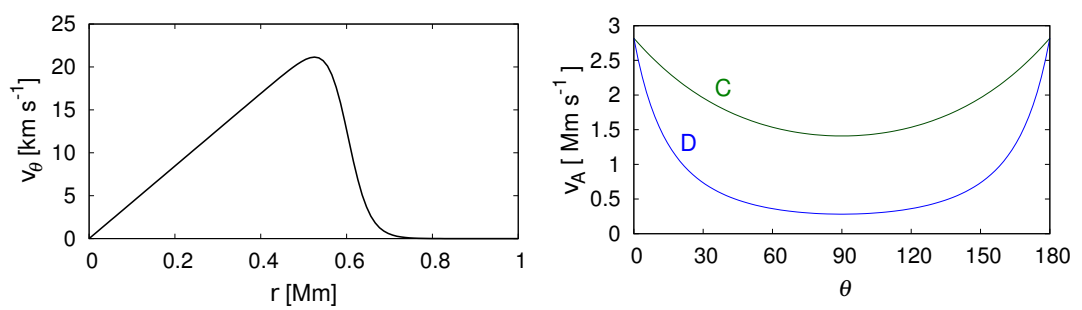

Figure 2. Left, the footpoint driving function for $0.1 t_{\mathrm{tw}} \lesssim t \lesssim 0.9 t_{t w}$. Right, the Alfvén speed along the central field line of loops $\mathrm{C}$ (green) and $\widetilde{D}$ (blue) $-\theta=90$ is the position at the loop apex.

Loops $\mathrm{C}$ and $\mathrm{D}$ have more realistic configurations, featuring large-scale curvature and are orientated differently compared to the straight loops. The curved loop domains have dimensions $x= \pm 10 \mathrm{Mm}, y= \pm 10 \mathrm{Mm}, z=0 \mathrm{Mm}-20 \mathrm{Mm}$, with both footpoints residing at the $z=0$ boundary, representing the chromosphere. Loop $\mathrm{C}$ is constructed such that the field converges towards the footpoints in a manner comparable to loop B (both have $B_{\mathrm{ftp}} / B_{\mathrm{apx}}=2$ ): the differences in results between these two loops should therefore reveal the impact of curvature. The footpoint positions for loop D are the same as those for loop C, and so these two loops exhibit a similar level of curvature; however, loop D is given five times the level of footpoint convergence $\left(B_{\mathrm{ftp}} / B_{\mathrm{apx}}=10\right)$ : the intention here is to continue to explore the impact of field convergence, but within the context of loop curvature.

The initial field for loop A is uniform and has a $z$-component only, while in loops $\mathrm{B}, \mathrm{C}$, and D the initial field is constructed using two point sources located 
outside the model domain:

$$
\boldsymbol{B}(\boldsymbol{r})=B_{\mathrm{s}}\left(\frac{\boldsymbol{r}-\boldsymbol{s}_{1}}{\left|\boldsymbol{r}-\boldsymbol{s}_{1}\right|^{3}}-\frac{\boldsymbol{r}-\boldsymbol{s}_{2}}{\left|\boldsymbol{r}-\boldsymbol{s}_{2}\right|^{3}}\right),
$$

where $\boldsymbol{r}$ is a position vector within the simulation volume, $s_{1}$ and $s_{2}$ are the positive and negative point sources; $B_{\mathrm{s}}$ is a simple scaling constant and is given a specific value such that the axial field strength at the footpoints is some multiple of the field strength at the apex. In model B these point sources are located outside the $z_{\min }$ and $z_{\max }$ boundaries, while in $\mathrm{C}$ and $\mathrm{D}$ they are both located below $z_{\min }$ boundary. Table 1 gives the point source coordinates and scaling factors required to implement the converged field geometries.

Table 1. The point source coordinates and scaling terms required for field convergence (see Equation (1)).

\begin{tabular}{cccccc}
\hline Loop & $\boldsymbol{s}_{1}$ & $\boldsymbol{s}_{2}$ & $B_{\mathrm{s}}$ & $B_{\mathrm{ftp}}$ & $B_{\mathrm{apx}}$ \\
\hline $\mathbf{A}$ & $\mathrm{n} / \mathrm{a}$ & $\mathrm{n} / \mathrm{a}$ & $\mathrm{n} / \mathrm{a}$ & 1 & 1 \\
$\mathbf{B}$ & $(0,0,-21.4)$ & $(0,0,21.4)$ & 114 & 1 & 0.5 \\
$\mathbf{C}$ & $(0,-7.5,-27.1)$ & $(0,7.5,-27.1)$ & 1480 & 1 & 0.5 \\
$\mathbf{D}$ & $(0,-7.5,-4.2)$ & $(0,7.5,-4.2)$ & 17.9 & 1 & 0.1 \\
\hline
\end{tabular}

The density and temperature are initially uniform in models A, B, C and D: $n=1.2 \times 10^{15} \mathrm{~m}^{-3}$ and $T=4 \times 10^{3} \mathrm{~K}$. The initial temperature of $4000 \mathrm{~K}$ is rather low even for the photosphere, let alone even hotter chromosphere and the corona. However, this temperature does not affect the evolution and thermodynamics of the fluxtubes, provided the temperature in heated regions is much higher. In addition, model $\mathrm{D}^{*}$ has a gravitationally stratified atmosphere, with more realistic temperature and density distributions (see Section 4.6).

For all loops discussed in this paper, the magnetic field is under the line-tied condition $(\eta=0$, i.e. $\partial \boldsymbol{B} / \partial t=\mathbf{0}$ when $\boldsymbol{v}=\mathbf{0})$ at the "foot-point" boundaries. Furthermore, all loop simulations begin with a potential field. The magnetic twist necessary for instability is created by rotating the plasma located at the footpoints; rotation vortices are, for the curved loops, centered at $x=0, y= \pm 7.5$, $z=0$. The rotational driving varies in space and time (Figure 2, left):

$$
v_{\theta}(r, t)=r \omega_{0} \frac{1-\tanh \left(\frac{r-r_{\mathrm{fr}}}{r_{\mathrm{fb}}}\right)}{2} \times \tanh \left(\frac{t}{t_{\mathrm{sw}}}\right) \times \frac{1-\tanh \left(\frac{t-t_{\mathrm{tw}}}{t_{\mathrm{sw}}}\right)}{2},
$$

where $r$ is the radial distance from the vortex centre, $\omega_{0}=0.015$ is a scaling factor, $r_{\mathrm{fr}}=0.6 R_{0}$ is the footpoint radius, $r_{\mathrm{fb}}=0.05 R_{0}$ is the footpoint boundary thickness, $t_{\mathrm{sw}}=20 t_{\mathrm{A}}$ is the switching time, and $t_{\mathrm{tw}}$ is the characteristic twisting time. There is a slightly different arrangement for the straight loops: the vortex 
centres are located at $(0,0, \pm 10)$ and the two footpoints are driven in opposite directions. Note, the dimensionalising factors are $R_{0}=1 \mathrm{Mm}$ and $t_{\mathrm{A}}=0.35 \mathrm{~s}$ (see Section 3 for further details). The important parameters are $\omega_{0}$ and $t_{\mathrm{tw}}$ : the product of these two terms, multiplied by two, gives the total twist after footpoint rotation has stopped: e.g., for $t_{\mathrm{tw}}=750$ the twist is $\approx 6 \pi$. Each loop is twisted for a time sufficient to cause an instability. The Poynting flux generated by the driving adds energy to the field; if the driving is continued through the unstable phase it becomes difficult to assess the energy released as the loop relaxes to a lower energy state. The twisting phase is purely "technical", i.e. it does not represent any real physical effects in the corona. (Indeed, vortical motions are often observed in the solar atmosphere, but they are much slower, with periods of at least tens of hours.)

The level of field convergence determines how the Alfvén speed varies with height. For loop A there is no convergence and $v_{\mathrm{A}} \approx 2800 \mathrm{~km} \mathrm{~s}^{-1}$ for all $z$, which is also true for the footpoints of loops B-D. Hence, the peak driving velocity $\left(\approx 21 \mathrm{~km} \mathrm{~s}^{-1}\right)$ is sub-Alfvénic (Figure 2 , right) for all four loops.

\section{Numerical Code and Simulation Setup}

All numerical simulations were performed using LARE3D - a three-dimensional Lagrangian remap MHD code (Arber et al., 2001). This code is based on a Lagrangian remap scheme: the Lagrangian part, which is done using a secondorder accurate predictor-corrector method, deforms the grid such that it moves with the plasma. The advantage of this technique is that additional physics, such as thermal conduction and shock capturing, can easily be incorporated into the code. The remap stage involves the mapping of the plasma properties (e.g., density, velocity, magnetic field) back to the original Cartesian grid; monotonicity is preserved through the use of Van Leer (1997) gradient limiters.

LARE3D solves the following resistive MHD equations,

$$
\begin{aligned}
\frac{\partial \rho}{\partial t} & =-\nabla \cdot(\rho \boldsymbol{v}), \\
\frac{\partial}{\partial t}(\rho \boldsymbol{v}) & =-\nabla \cdot(\rho \boldsymbol{v} \boldsymbol{v})+\frac{1}{\mu_{0}}(\nabla \times \boldsymbol{B}) \times \boldsymbol{B}-\nabla P-\rho g \boldsymbol{z}+\nabla \cdot \sigma \\
\frac{\partial \boldsymbol{B}}{\partial t} & =\nabla \times(\boldsymbol{v} \times \boldsymbol{B})-\nabla \times\left(\eta \frac{\nabla \times \boldsymbol{B}}{\mu_{0}}\right), \\
\frac{\partial}{\partial t}(\rho \epsilon) & =-\nabla \cdot(\rho \epsilon \boldsymbol{v})-P \nabla \cdot \boldsymbol{v}+\eta J^{2}+\nabla \cdot \boldsymbol{q}+\varepsilon \sigma,
\end{aligned}
$$

with specific energy density

$$
\epsilon=\frac{P}{(\gamma-1) \rho}
$$


and current density

$$
\boldsymbol{J}=\frac{1}{\mu_{0}} \nabla \times \boldsymbol{B},
$$

where $\rho$ is the mass density, $\boldsymbol{v}$ is the plasma velocity, $\boldsymbol{B}$ the magnetic field, $P$ the thermal pressure, $\eta$ is the resistivity, $\gamma=5 / 3$ is the ratio of specific heats, $\mu_{0}$ is the magnetic permeability, and $\boldsymbol{q}$ is the conductive heat flux. Radiation is ignored in this study, but it is not expected to have a significant impact, since the radiative timescale is much longer than the magnetic relaxation timescale. The gravity is ignored $(g=0)$ in models with uniform atmosphere (A-D), but included in model $\mathrm{D}^{*}$ with atmospheric stratification. The last terms of equations 4 and 6 feature tensors, and are required to simulate shock heating; these terms are defined in the last paragraph of this section.

The magnetic field is made line-tied simply by setting the plasma fully ideal at the lower boundary, $\eta(z=0)=0$. The specific energy and the density at the lower boundary (and slightly below, in the 'ghost cells' (see Arber et al., 2001) are fixed and either constant (in loops A-D) or correspond to the gravitationally stratified atmosphere (in loop $\mathrm{D}^{*}$ ), as defined in the Sect. 4.6. All variables at the upper and side boundaries are set as for the 'free' boundaries, i.e. their normal gradients are set to zero, $\frac{\partial}{\partial \boldsymbol{n}}=0$. This type of boundary conditions results in a stable solution with almost no reflections from the upper and lower boundaries, so that any perturbation reaching one of these boundaries leaves the domain.

We normalise the variables in the MHD equations using reference values suitable for a coronal active region:

$$
r=\frac{r^{*}}{R_{0}}, \quad \rho=\frac{\rho^{*}}{\rho_{0}}, \quad B=\frac{B^{*}}{B_{0}},
$$

where asterisks denote the unnormalised MHD variables, $R_{0}=1 \mathrm{Mm}, \rho_{0}=$ $2 \times 10^{-12} \mathrm{~kg} \mathrm{~m}^{-3}$ and $B_{0}=4.47 \times 10^{-3} \mathrm{~T}$. Other variables are expressed as

$$
L=\frac{L^{*}}{R_{0}}, \quad t=\frac{t^{*}}{t_{\mathrm{A}}}, \quad v=\frac{v^{*}}{v_{\mathrm{A}}}, \quad P=\frac{P^{*}}{P_{0}},
$$

where $v_{A}=B_{0} / \sqrt{\mu_{0} \rho_{0}}$ is the reference Alfvén speed, $t_{\mathrm{A}}=R_{0} / v_{\mathrm{A}}$ is the reference Alfvén time, and $P_{0}=B_{0}^{2} / \mu_{0}$ is the reference pressure. The specific energy density, current density, and resistivity $(\epsilon, J$, and $\eta)$ also have reference variables that can be expressed in terms of $R_{0}, \rho_{0}$ and $B_{0}$ :

$$
\epsilon_{0}=\frac{B_{0}^{2}}{\mu_{0} \rho_{0}}=v_{\mathrm{A}}^{2}, \quad J_{0}=\frac{B_{0}}{\mu_{0} R_{0}}, \quad \eta_{0}=\mu_{0} R_{0} v_{\mathrm{A}} .
$$

Hence, for the chosen values of $R_{0}, \rho_{0}$ and $B_{0}, t_{\mathrm{A}} \approx 0.36 \mathrm{~s}, v_{\mathrm{A}} \approx 2800 \mathrm{~km} \mathrm{~s}^{-1}$ and $\eta_{0} \approx 1.1 \pi \times 10^{6} \Omega \mathrm{m}$.

In all four cases, the simulation features two stages (see also Gordovskyy et al., 2013): twisting until the fluxtube becomes kink-unstable and magnetic relaxation after the kink instability. The first stage is done using ideal MHD: Ohmic dissipation and conduction are absent from the energy equation. The 
footpoint driving eventually induces a kink instability that initiates a release of magnetic energy. Just before this point, the simulations are restarted with the resistive and conductive terms now included in the MHD equations. Any heating will reduce density and thereby increase the local Alfvén speed, which then leads to a decrease in the time step under the CFL condition. For the converged loops the magnetic field is strongest at the footpoints, and so the driving will inevitably create the highest currents at these locations. The burst of Ohmic heating caused by the switch on of resistive MHD can be so intense as to cause cavitation at the $z$-boundaries; the result is that the time step becomes too small for the simulation to make progress. Fortunately, this issue can be avoided through the use of thermal conduction, assuming that the plasma below the footpoints is maintained at a constant cold temperature equivalent to $4000 \mathrm{~K}$.

Throughout the loop volume there is a background resistivity of $\eta_{\mathrm{b}}=3 \times 10^{-7}$, except when the current reaches or exceeds a threshold $\left(j_{\text {crit }}=2\right)$, at which point an anomalous resistivity, $\eta_{\mathrm{c}}=0.002$, is applied. The lower the value of $j_{\text {crit }}$ the greater the percentage of the loop interior that will be assigned an anomalous resistivity when the simulation is restarted in resistive mode. We chose a current threshold of two, since this results in $10 \%$ of loop A contributing to Ohmic heating immediately after the restart.

The computational domain is a $3 \mathrm{D}$ staggered grid: physical variables are not calculated at the same place for each cell in the domain, which improves numerical stability and allows conservation laws to be included in the computation. There are some differences between the straight and curved loops as regards grid dimensions and limits. The straight loop simulations are run at a grid resolution of $128^{2} \times 256$, whereas for the curved loops it is $256^{2} \times 512$. The differences in grid volume and resolution between the straight and curved loop simulations mean that, along the $x$ and $y$ axes, the straight loops are better resolved by a factor of two; however, the curved loops have double the resolution along the $z$ axis.

The conductive heat flux (Equation (6)) is implemented using Braginskii parallel conduction (Braginskii, 1965). It provides only parallel heat flux in the presence of non-zero magnetic field, and becomes isotropic where the magnetic field is nearly zero.

LARE3D uses shock viscosity (Wilkins, 1980) to capture the heating effect of shocks, this is represented by the terms $\nabla \cdot \sigma$ and $\epsilon \sigma$ in the main equations in Sect 3 . This effect does not represent a physical viscosity in the corona and is used to regularise the solution near shocks. However, it appears that this shock viscosity, in fact, can represent a real physical effect. Recently, Bareford and Hood (2015) conducted a detailed investigation of shock handling within LARE3D. They have shown that, for kink-unstable loop simulations, shock viscosity heating in LARE3D is consistent with Petschek reconnection and slow-mode shocks. Unlike the anomalous resistivity, controlled by the externally-defined critical current, the dissipation due to shock viscosity is defined internally, by the velocity field in the simulation domain. 

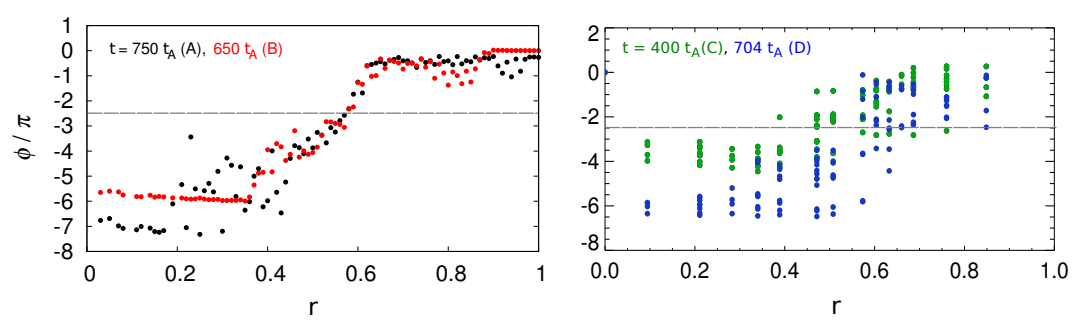

Figure 3. Left, the magnetic twist in units of $\pi$ as a function of radial distance from footpoint centre for the straight loops, A (black) and B (red). Right, the same plots but for the curved loops, C (green) and D (blue). The twist values were determined numerically just before instability onset, see time labels. The twist threshold for an ideal kink instability $(\phi \approx 2.49 \pi)$ as calculated by Hood and Priest (1979) is given by the dashed horizontal line.
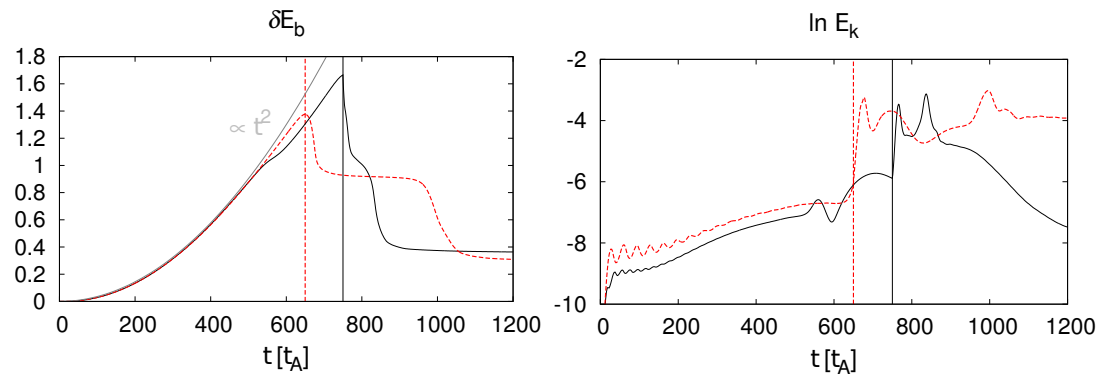

Figure 4. The change in volume-integrated magnetic energy (left) and the natural logarithm of the kinetic energy (right) for loop A (black solid line, arbitrary units) and for loop B (red dashed line). Resistive MHD was switched on at $t=750 t_{\mathrm{A}}$ for loop A and $t=650 t_{\mathrm{A}}$ for loop $\mathrm{B}$, i.e., just before instability. Vertical lines show the onset of kink-instability. The initial magnetic energies are $E_{\mathrm{b}}=250$ for loop A and $E_{\mathrm{b}}=103$ for loop B. Peak kinetic energies are 0.0045 for loop A and 0.05 for loop B.
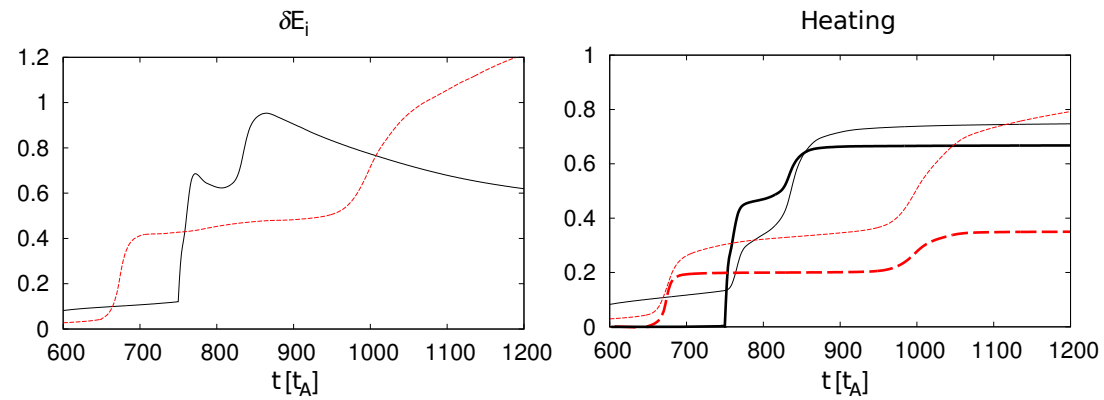

Figure 5. Left: the volume-integrated change in internal energy. Right: the cumulative Ohmic heating (thick lines) and shock heating (thin lines). Black solid lines are for loop A and red dashed lines are for loop B.

\section{Results of Numerical Simulations}

This section presents the results taken from the loop simulations (A-D and $\left.\mathrm{D}^{*}\right)$. Some properties are given in SI units; these are length, temperature and density. 
Velocities are expressed in either $\mathrm{km} \mathrm{s}^{-1}$ or $\mathrm{Mms}^{-1}$. Times are given in units of the Alfvén time, whereas energies and currents are given in normalised units.

The twist angles before the kink-instabilities for models A-D are shown in Figure 3. Total magnetic, kinetic and internal energies, and cumulative Ohmic and shock heatings for models A and B are shown in Figures 4 and 5. Current densities and temperature distributions for models $\mathrm{A}$ and $\mathrm{B}$ are shown in Figures 6 and 7, respectively. The distributions of anomalous resistivity in models A and $\mathrm{B}$ are shown in Figure 8. Plasma velocities along the fluxtube axis in models $\mathrm{A}$ and $\mathrm{B}$ are shown in Figure 9. Total magnetic, kinetic and internal energies, and cumulative Ohmic and shock heatings for models $\mathrm{C}$ and $\mathrm{D}$ are shown in Figures 10 and 11. The current density distributions for models C and D are shown in Figure 12, while the temperature distributions for these models are shown in Figures 13 and 14. Figure 15 demonstrates location and a structure of a typical reconnection region in a twisted loop. Finally, Figures 16, 17 and 18 show the initial atmosphere configuration, total energies and critical twist for model $D^{*}$, respectively.

Let us now consider how the twisted fluxtube parameters affect their stability, and the energy release process.

\subsection{Kink Instability, Magnetic Reconnection and Energy Release}
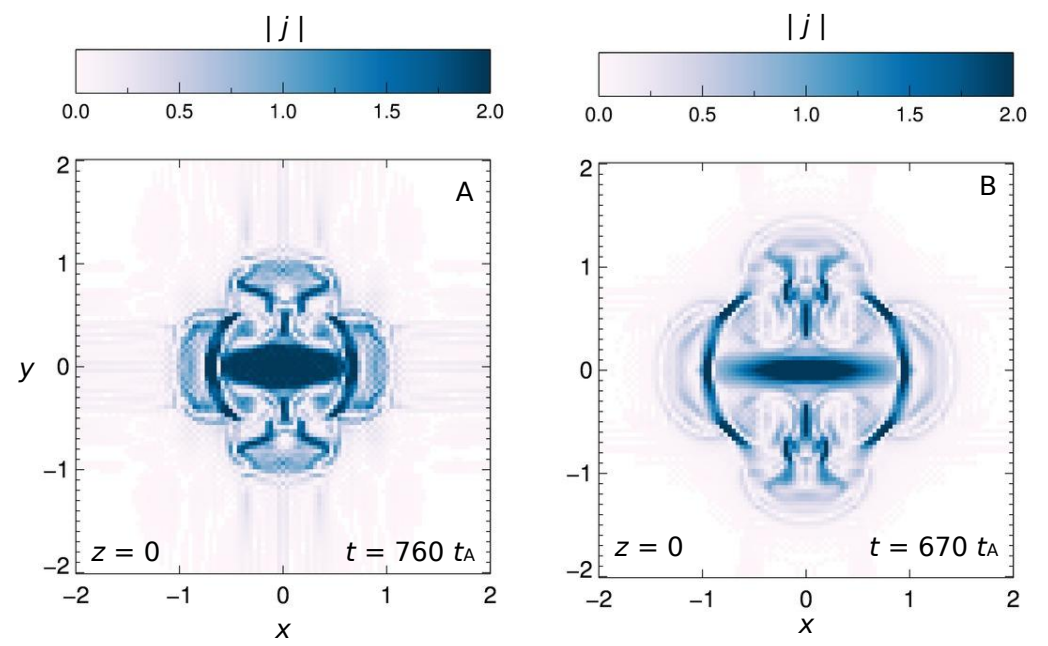

Figure 6. The current density magnitude (dark regions correspond to the highest currents) over the $x-y$ plane at $z=0$ shortly after instability onset for loops A (left) and $\mathrm{B}$ (right).

The helicity is injected by rotating footpoints of initially potential fluxtubes (see Section 2). When a critical level of twist is reached in each fluxtube, they become unstable, leading to current sheet formation and energy release. The nature of this process is discussed in more detail in Section 4.5.

Here we are interested in the following: what is the twist angle required for the kink-instability, how much energy is released, what is the spatial distribution of 

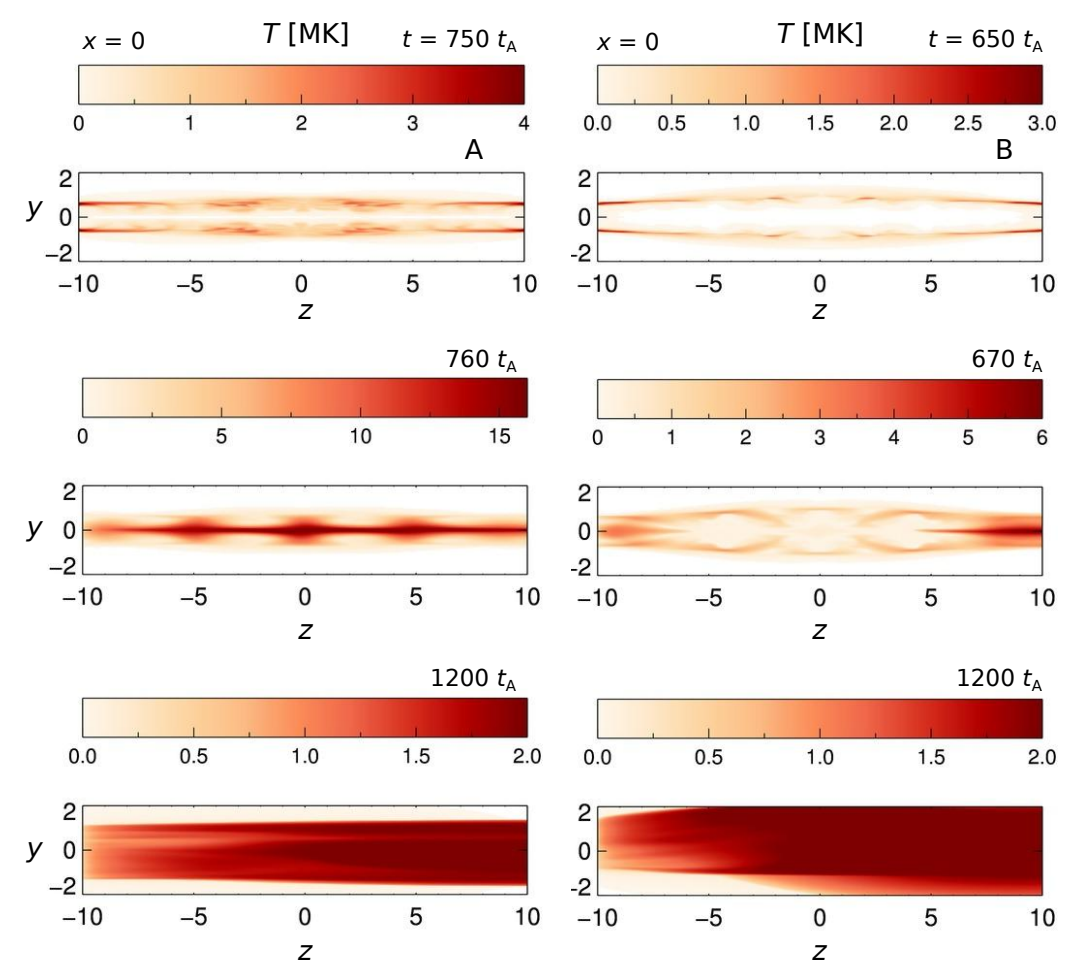

Figure 7. The temperature over the $y-z$ plane $(x=0)$ at times $t=750$ (top), 760 (middle) and $1200 t_{\mathrm{A}}$ (bottom) for loop A (left), and for loop B (right).

the heated plasma, and how there characteristics depend on loop configuration and ambient atmosphere parameters?

First, let us consider the twist angle just before the kink instability. For a cylindrically symmetric twisted fluxtube, the total twist $\Phi$ is the angle described by a magnetic field line around the central axis from one foot-point to another. In terms of the magnetic field components, it can be expressed as $\Phi=\frac{\mathcal{L} B_{\theta}}{r B_{z}}$, where $\mathcal{L}$ is the loop length, $r$ is the radial distance between the field line and the axis (it is assumed that each field line lies on a cylindrical surface), $B_{\theta}$ and $B_{z}$ are the azimuthal and longitudinal field components, respectively. However, when the fluxtube is not cylindrically symmetric, measuring this angle is not straightforward. Figure 3 is created by following field lines from specific points on the postive footpoint. The starting points are distinguished by the distance from the footpoint centre, located at $(0,0,-10)$ for the straight loops and $(0,-7.5,0)$ for the curved; and the magnetic twist is calculated numerically by keeping track of how many times a field line wraps around the initial loop axis before it reaches the negative footpoint. The twist measurements were taken at a time close to instability onset. It can be clearly seen that the level of twist for both straight fluxtubes A and B, and loops C, D and D* exceeds $2.49 \pi$, the result obtained by Hood and Priest (1979) which is applicable to a straight loop of aspect ratio ten $(=L / R)$ : hence, this value should only be treated as a necessary condition for instability. At radii less than 0.4 , the magnetic twist is about $7 \pi$ in fluxtube 
A and about $5.5-6 \pi$ in fluxtube B. This is consistent with the average twist on the instability threshold (where the twist is single-signed) reported by Bareford et al. (2011). However, the curved loops are somewhat less twisted: in particular, the twist of magnetic field in loop $\mathrm{D}$ is about $6 \pi$ at $r \lesssim 0.4$, while in the loop $\mathrm{C}$ it is even lower, around 4-4.5 $\pi$. Finally, loop $\mathrm{D}^{*}$ (strongly converging loop in stratified atmosphere, see Figure 18) has the critical twist value of about $4-4.5 \pi$.

Let us discuss the evolution of the magnetic energy in the considered loops. The volume integrated magnetic energy plots are shown in Figures 4, 10 and 17. It can be seen that the energy increase during the twisting phase in loops A and $\mathrm{B}$ is nearly identical as expected, since the initial magnetic field and rotation at footpoints are the same. However, in loop $\mathrm{C}$ the energy increases more slowly compared to the loop D. This is most likely because of the different magnetic field distribution in the foot-points of the loop $\mathrm{C}$ and, most importantly, stronger field inclination, which, in turns, results in a difference between Poynting fluxes in loops $\mathrm{C}$ and $\mathrm{D}$.

Variation of the magnetic energy after the instability is qualitatively similar in all models. The only substantial difference is that in loops A and B the energy is released in two impulses. This effect is most prominent in loop B, where these impulses are separated by about $250 t_{\mathrm{A}}$. It is common for energy release to proceed somewhat unevenly, with a series of "spikes" in kinetic energy - but loops A and B, unusually, show a two-phase relaxation in which the reconnection seems to stall and then re-start. This is very likely because this loop is driven only until it is just over the stability threshold - and indeed, as mentioned earlier in Section 4.3, this feature disappears if the loop is driven for longer. Another reason could be that, due to the convergence, reconnection in loop B takes place in different parts of the loop at different times.

As far as the curved loops are concerned, they are noticeably different in how fast the magnetic energy drops after the instability. Loop $\mathrm{C}$ is consistent with the kink-like instabilities seen for the straight loops: there is a swift drop in magnetic energy coincident with rises in heating (both Ohmic and shock) and internal energy. Furthermore, the unstable phase is also accompanied by peaks in kinetic energy. Loop D on the other hand, shows a more gradual drop in magnetic energy (Figure 10), which does not correspond to the rise in Ohmic heating; instead the similarly gradual increase in internal energy is caused by shock heating. Surprisingly, the kinetic energy remains high even as loop D settles into a lower energy state. Driving loop $\mathrm{C}$ for the same amount of time as loop $\mathrm{D}$ (i.e., $t_{\mathrm{tw}} \approx 600 t_{\mathrm{A}}$ ) does little to alter how the magnetic energy changes during the simulation. The instability occurs at roughly the same time as before $\left(400 t_{\mathrm{A}}\right)$, but the energy released is halved: the continued driving replenishes the energy, while it still dissipates, and interferes with how the instability plays out.

The left panel of Figure 10 shows that very strong footpoint convergence almost completely cancels the effect of curvature. The fall in magnetic energy still happens earlier than for the straight loops, but the growth in magnetic energy before instability far outstrips that seen in the other simulations. The normalised increase in magnetic energy is eight times higher than that for loop B.

We now turn our attention to showing whether or not the results from loop $\mathrm{D}$ are indeed consistent with a kink instability. Although, Figure 3 (right) shows 
that loop D is sufficiently twisted (stronger than loop C), we also see that the changes in magnetic energy and the kinetic energy logarithm (Figure 10, blue lines) are somewhat different from the forms seen for other kink-unstable loops. Specifically, the decline in magnetic energy is noticeably slower and not accompanied by a rapid change in kinetic energy. It seems that increasing the convergence (compared to loop $\mathrm{C}$ ) has prolonged the transition to a low-energy state.

However, the magnetic fieldline plots in Figures 13 and 14 (right columns) do agree with the form expected for a kink instability: initially, the fieldlines are tightly twisted around the loop axis, and then, as the instability proceeds, the fieldlines untwist. Loop $\mathrm{C}$ undergoes an internal kink instability, since the apex height $(z \approx 3.8)$ remains almost constant throughout the simulation, and the heating (a mixture of Ohmic and shock) is concentrated around the apex during the unstable phase. As the loop relaxes, conduction combined with continued heating results in a near-uniform temperature of around $1.5 \mathrm{MK}$. The temperature plots for loop D do suggest an increase in apex height. On the other hand, the corresponding fieldline plots indicate that the apex rise is temporary and is no longer evident once the loop has relaxed. Figure 14 also shows that the (mostly shock) heating is concentrated along the legs of the loop, and, for the scaling used here $\left(B_{0}=44.7 \mathrm{G}\right)$, results in sub-MK temperatures. There is some Ohmic heating at instability onset $\left(t=600 t_{\mathrm{A}}\right)$, but it is confined to the footpoints.

\subsection{Ohmic heating versus shock viscosity dissipation}
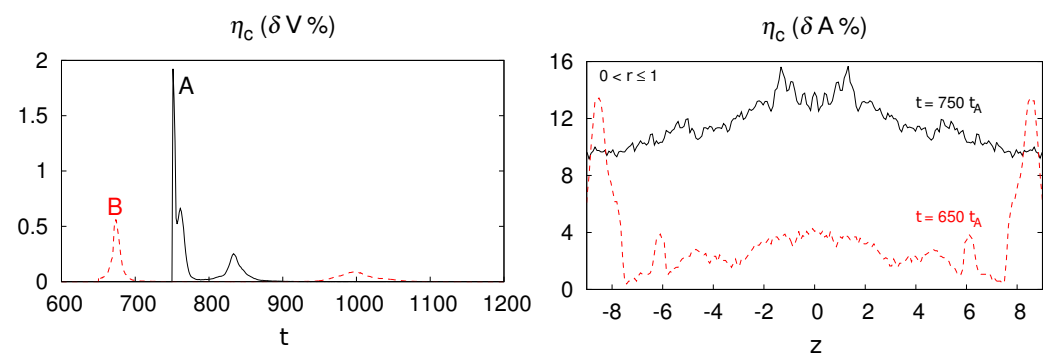

Figure 8. Left, the percentage of the grid assigned anomalous resistivity for loop A (black solid lines) and for loop B (red dashed lines). Right, the percentage of the cross sectional area $(0<r \leq 1)$ assigned anomalous resistivity at instability onset,

Comparison of all four models shows that the ratio of viscous to Ohmic dissipation changes from about 1 for a non-converging fluxtube (see also Hood et al., 2009) to as much as $\approx 10$ in the strongly converging loop in model D. While the contribution of the Ohmic and viscous heating in models A and B is nearly uniform along the fluxtubes, in curved fluxtubes in models $\mathrm{C}$ and $\mathrm{D}$ the Ohmic heating is prevalent very close to footpoints (where the current density is high), while in the middle of the loops, where velocitites and velocity gradients are high, the magnetic energy dissipates through the "viscosity" channel. Hence, the enhanced viscous dissipation in converging fluxtubes is most likely caused 

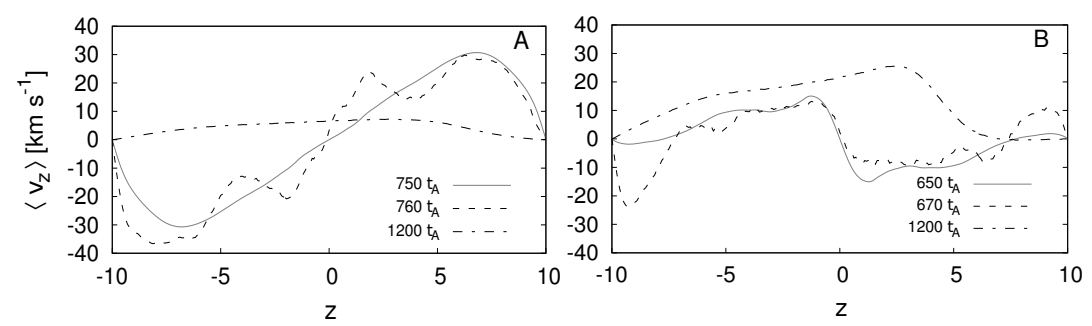

Figure 9. The average velocity parallel to the $z$-axis at three times for loops A (left) and $\mathrm{B}$ (right). The velocity component $v_{\mathrm{z}}$ is averaged over $0<r \leq 0.8$ for each $z$ coordinate.

by shock formation due to low magnetic field and, hence, low Alfvén velocities near the fluxtube centres.

Strong shock dissipation in model D can explain the delay of the kink instability. Since the shock viscosity effectively acts as an additional dissipation channel for magnetic energy (see Section 3), the rates of helicity injection and magnetic energy accumulation (due to the footpoint rotation) are lower. However, as far as the reconnection stage is concerned, the slower energy release is mainly due to the field convergence: lower magnetic field near the looptop and, hence, lower current densities, reduce the Ohmic heating rate, which is proportional to $J^{2}$.

The ratio of Ohmic to shock viscosity heating varies with time. Shock viscosity heating normally dominates, and it seems to become even stronger on later stages of the relaxation process. This may be explained by the development of shocks and other small-scale details in the velocity field. The latter can be seen in the velocity data. Thus, Figure 9 shows how $v_{\mathrm{z}}$ varies along the loop axis. The velocity is averaged over the loop cross-section $(0<r \leq 0.8)$ for the same times as the temperature plots. Before the instability (solid line), the stongest axial flows for loop A are away from the apex. These flows diminish as the instability progresses, resulting in a residual left to right flow by the time loop A has relaxed. Loop B has a more complicated flow pattern, consistent with heating sources located at $z= \pm 7.5$. Over time the flow becomes chaotic, but the end result is similar to loop A, albeit with a higher rightward flow.

\subsection{Footpoint Convergence}

In order to investigate the role of footpoint convergence we firstly compare the results for loops $\mathrm{A}$ and $\mathrm{B}$. The former begins with a uniform straight field of $|\boldsymbol{B}|=1$, whereas the latter begins with a field that has less energy, since $|\boldsymbol{B}|$ only rises to one at the footpoints.

Figures 4 and 5 present the changes in volume-integrated energies. The vortical footpoint driving (Equation (2)) leads to instability for both cases; however, the converged straight loop (B) is the first to achieve instability. For this reason, the driving is terminated sooner and resistive MHD is switched on earlier, see the vertical dash lines in Figure 4. It is important to note, that in fluxtubes A and $\mathrm{B}$ the twist angle and the total magnetic energy increase with time at the same rate, since their normal footpoint magnetic field $\left(B_{\mathrm{z}}\right)$ distributions - and, 

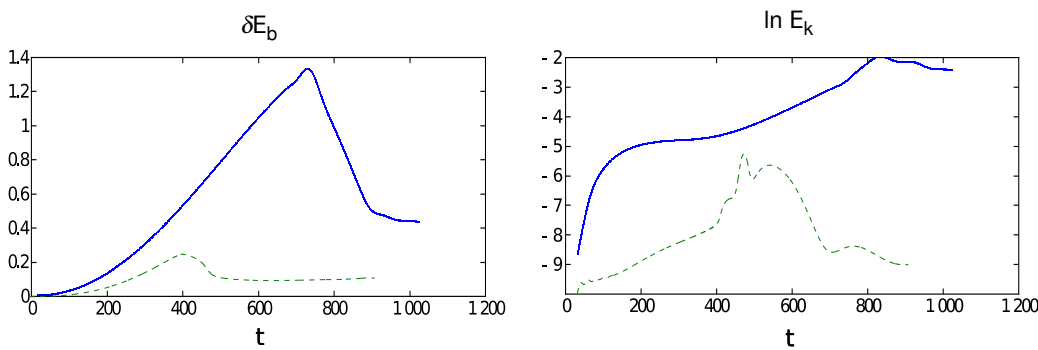

Figure 10. The change in volume-integrated magnetic energy (left) and the natural logarithm of the kinetic energy (right, arbitrary units) for loop C (green dashed lines) and for loop D (blue solid lines). Resistive MHD was switched on at $t=400 t_{\mathrm{A}}$ for loop $\mathrm{C}$ and $t=600 t_{\mathrm{A}}$ for loop $\mathrm{D}$, i.e., just before instability. The initial magnetic energies are 980 and 43 for loops C and D, respectively. Peak kinetic energies are 0.05 and 0.42 for loops $\mathrm{C}$ and $\mathrm{D}$, respectively.
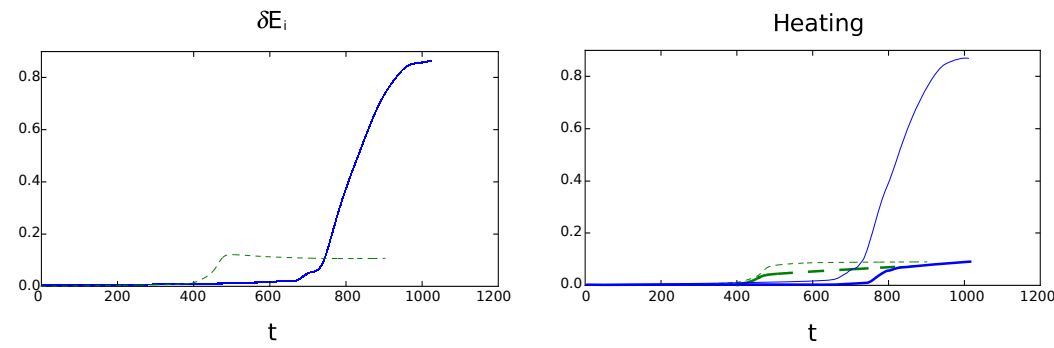

Figure 11. The change in volume-integrated internal energy (left) and the cumulative Ohmic and shock heating (right) for loop C (green dashed lines) and for loop D (blue solid lines). Thick and thin lines in the right panel correspond to Ohmic and shock heating, respectively.

hence, the Poynting fluxes from the photosphere - are nearly the same. However, loops $\mathrm{C}$ and $\mathrm{D}$ show different rates of twist and energy increase: although the amplitude of $B_{\mathrm{z}}$ at their footpoints is again the same, the strong field inclination in loop $\mathrm{C}$ along with much lower field convergence (compared to loop D), result in different Poynting fluxes for the same angular speed of footpoint rotation (compare Figures 3, 4, 10). Consequently, loop B has less azimuthal field prior to the unstable phase.

Interestingly, after $t=650 t_{\mathrm{A}}$, loop B undergoes two bursts of energy release. The first occurs, as expected, at the end of the driving phase, then the loop stabilises for $250 t_{\mathrm{A}}$ before undergoing a slightly greater burst of energy release (similarly, loop A shows a quasi-stable period centred on $800 t_{\mathrm{A}}$ ). Another consequence of loop B being less stable is that less energy is released, since instability was reached after a shorter period of driving. Incidentally, the energy released by loop B increases only marginally $(\approx 8 \%)$ should the driving phase be extended to $t=750 t_{\mathrm{A}}$ (as is the case with loop $\mathrm{A}$ ): the instability is delayed until this later time after which the magnetic energy declines to a relaxed state, with no intervening stable period. 

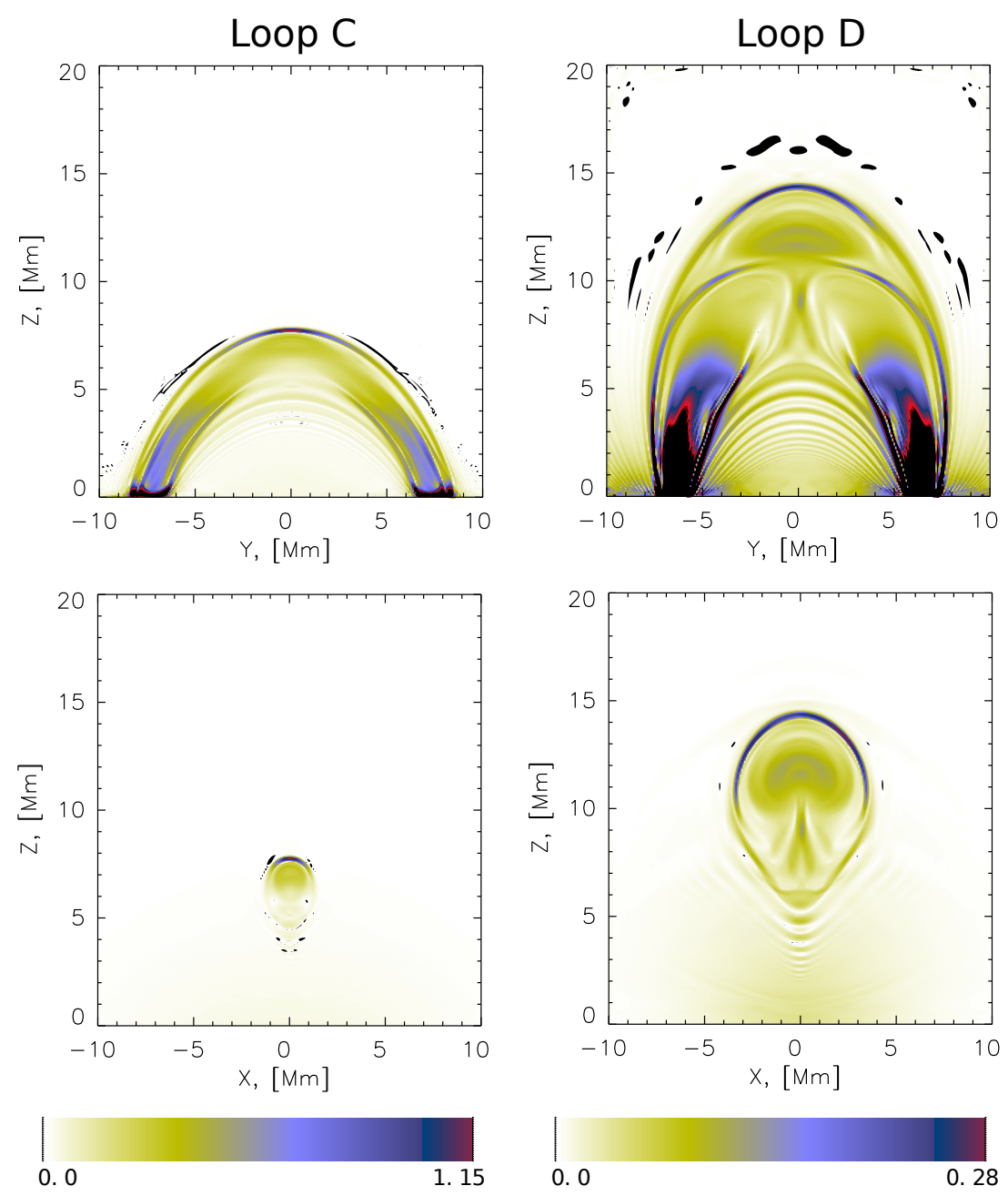

Figure 12. Current density distributions just before the kink instability in models $\mathrm{C}$ and D. Upper panels show loop mid-planes $(x=0)$, lower panels show central crosssections $(y=0)$. Current density scales are shown in units of $j_{0}=3.6 \times 10^{-3} \mathrm{~A} \mathrm{~m}^{-2}$.

The effect of magnetic field convergence on stability is rather complex. Thus, the weakly converging fluxtube B is less stable (i.e., requires lower twist angle for a kink-instability) than non-converging fluxtube A. On the other hand, strongly converging loop D is more stable than weakly converging loop C. This is because there are a few competing factors that could determine the stability of a twisted fluxtube.

Firstly, twisted fluxtubes with stronger convergence (and the same crosssection at footpoints) have, obviously, larger average radius. It has been shown (using linear MHD calculations) that fluxtubes with larger cross-section (and the same footpoints and length) are normally more stable (Browning and Van der Linden, 2003; Bareford et al., 2011). This is, most likely, the reason why strongly 

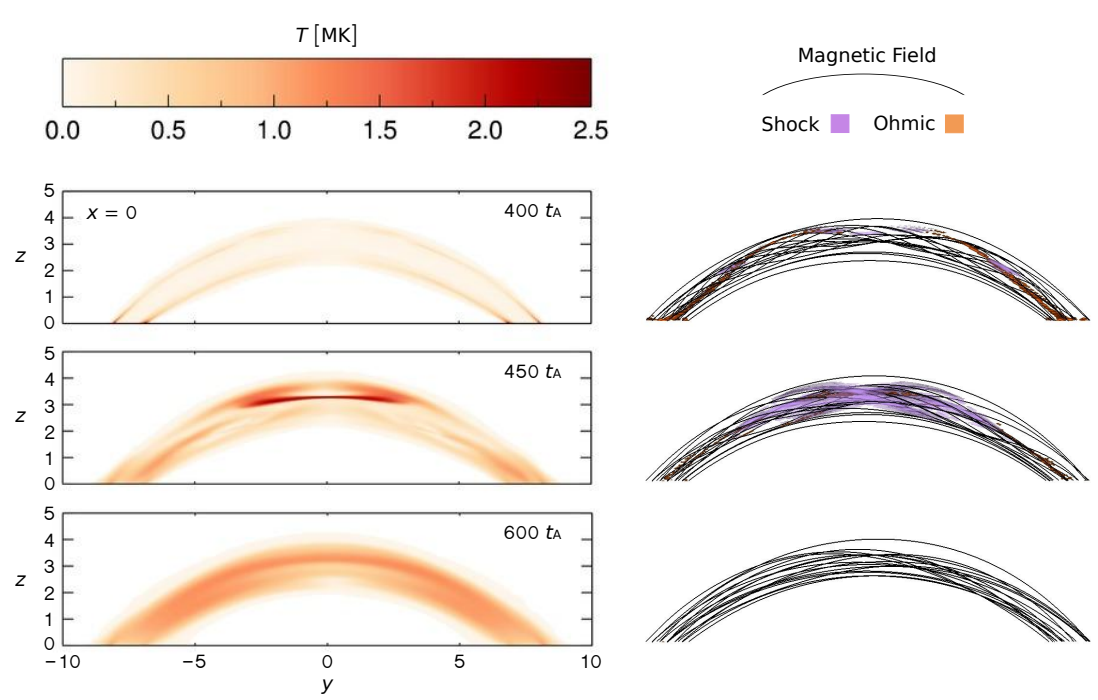

Figure 13. The temperature distribution in the mid-plane of the Loop C (left column) taken at three times, instability onset (top), during the instability (middle) and the relaxed state (bottom). The configuration of the field lines, alongwith the pattern of Ohmic (orange) and shock (purple) heating (right column), are shown for the same times.

converging loop D is more stable than loop C. At the same time, twisted fluxtubes with stronger convergence have larger magnetic energy associated with the azimuthal field (i.e., volume-integrated value of $B_{\theta}^{2} /\left(2 \mu_{0}\right)$ ), and this is believed to be a destabilising factor. This is likely to be the reason why weakly converging fluxtube B is less stable than non-converging fluxtube A - the effect of magnetic energy density dominates here.

It is expected that the driving should generate a linear increase in azimuthal field over time: hence, the increase in magnetic energy should show a $t^{2}$ dependence. This relationship is confirmed for loop A by the line in Figure 4 (left). After the driving phase, the natural logarithm of the kinetic energy rises again when resistive MHD is switched on (the gradient of $\ln E_{\text {kin }}$ is twice that of $\gamma$, the growth rate of the instability, since the velocity of the perturbed plasma is proportional to $\mathrm{e}^{\gamma t}$ ). The wave-like forms present for both straight loops from the start of the simulations are a consequence of the fact that the driving speed is supersonic; however, by $t=400 t_{\mathrm{A}}$, these oscillations have diffused away, which indicates that the field is now evolving through a sequence of equilibria (Mellor et al., 2005). Overall, the numerical dissipation, measured as a percentage of the total initial energy, is $0.11 \%$ for both straight loops.

Spatial distribution of the magnetic energy release depends on the spatial structure of current density and resistivity.

Inevitably, the driving creates high currents at the footpoints - these are not shown in Figure 8 (right) in order to reveal how $\eta$ changes around the loop apex. Nevertheless, for loop A, $90 \%$ of the loop volume assigned anomalous resistivity occurs within $-9 \leq z \leq 9$, whereas loop B has $82 \%$ of the anomalous resistivity within this range. The levels of Ohmic and shock heating are roughly equal for 

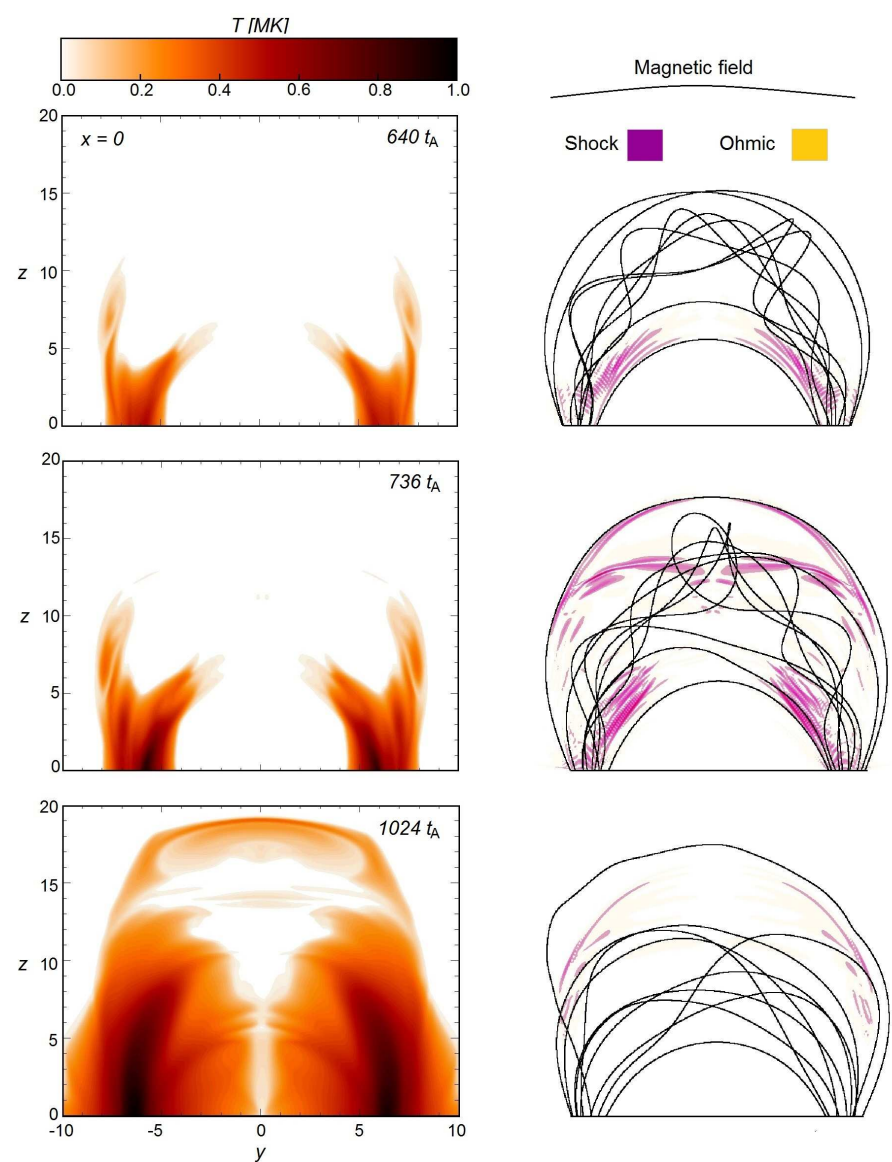

Figure 14. The same as in Figure 13, but for loop D. At $t=736 t_{\mathrm{A}}$, the temperature plot is saturated: near the footpoints, the highest temperatures are $6 \mathrm{MK}$.

the unconverged loop (A), but when the field is converged the shock heating becomes steadily greater as the simulation progresses: in fact, Ohmic heating only increases during the two periods of magnetic energy decline.

We now investigate the current structures existing shortly after instability onset (Figures 6 and 12). Similar to previous simulations of kink-unstable loops (Browning et al., 2008; Hood et al., 2009; Botha et al., 2011; Bareford et al., 2013), the current sheets in the cylindrical models A and B initially form a helical ribbon structure around the kinking fluxtubes. In addition, there is a strong currect along the fluxtube axis $(x=y=0)$. This current structure has elliptic cross-section with its main axis rotating along the $z$-axis; the total rotation angle just after the kink is approximately the same as the total magnetic twist angle. In the model A (with initially cylindrical fluxtube) the current density is nearly uniform along $z$-axis: it is slightly higher in the central region of the fluxtube (i.e., around $z=0$ ), while in the fluxtube with converging field (model $\mathrm{B}$ ) it 
also has high current densities near the footpoints (see also Gordovskyy and Browning, 2011, 2012).

Next, we examine how heat is distributed within the two straight loops at three times, $t=750 t_{\mathrm{A}}$ (instability onset), $760 t_{\mathrm{A}}$ (immediately after the instability) and $1200 t_{\mathrm{A}}$ (when the field has relaxed to a lower energy state), see Figure 7. Leading up to the instability (top row), hot plasma forms shells around the fluxtubes, apparently, corresponding to the current density concentrations. At this stage the temperatures are also comparable. However, immediately after the instability, temperatures for loop A increase by a factor of four, with the highest temperatures forming along the axis. The radial spread of heating at certain locations along the $z$-axis reveals where the loop has kinked. Contrastingly, loop B shows the strongest heating near the footpoints, as well as in the shell around the fluxtube; the temperatures are roughly double those before the instability. As the loops relax, thermal conduction acts to smooth out the temperatures, yielding an average value of $2 \mathrm{MK}$. Relatively low temperatures (less than $10 \mathrm{MK}$ ) are compatible with the temperatures observed in nanoflares (e.g. Brosius et al., 2014). The amount of released energy and peak temperature strongly depend on the magnetic energy density in the flaring loop. Thus, Gordovskyy et al. (2015) investigated loops with stronger magnetic field and reached much higher peak temperatures during the reconnection, up to $30 \mathrm{MK}$.

\subsection{Loop Curvature}

The obvious difference between curved loops (models C and D) and cylindrical fluxtubes is the geometry of current density distribution. In the straight loop (model A) currents are nearly uniformly distributed along the loop, while in the converging fluxtube (model B) the current density is, as one would expect, higher near the footpoints. However, in both models the structure has a high degree of cylindrical symmetry, unlike the curved loops, where, just before the instability occurs, the current is concentrated in a thin shield above the loop top (see Figure 12). In addition, there are current concentrations close to footpoints due to the field convergence.

Loop D has the most highly converged footpoint field, which means the field strength at the apex is the weakest (all loops have the same footpoint field strength). The result of this is that loop D has by far the smallest volumeintegrated field energy - over ten times smaller than the total field energy for the other curved loop $(\mathrm{C})$. Hence, the energy plots for these two models are strikingly different.

Comparing the rise in magnetic energy in our models, we note that curvature results is lower rates at which driving adds magnetic energy to the loop. At the same time however, large-scale curvature has made the loop more susceptible to instability: a loss of magnetic energy occurs much earlier. This is also clearly seen from the comparison of the critical twist angles: in curved loops $\mathrm{C}$ and $\mathrm{D}$ they are lower than in cylindrical fluxtubes A and B. Hence, the curvature exerts a destabilising influence: i.e., the average absolute twist at instability onset is lower than it is for the straight loops.

The reason for this effect is not immediately clear. One of the most obvious possible explanations is that the magnetic field in curved loops has some 
curvature of about $\pi$ from the beginning. This alone can explain the difference between the critical twist angles, which is around $\pi$ (see Figure 3). However, higher convergence near the footpoints and loss of symmetry in curved loops described above are also expected to reduce stability.

\subsection{Structure of Magnetic Reconnection in Twisted Fluxtube Models}
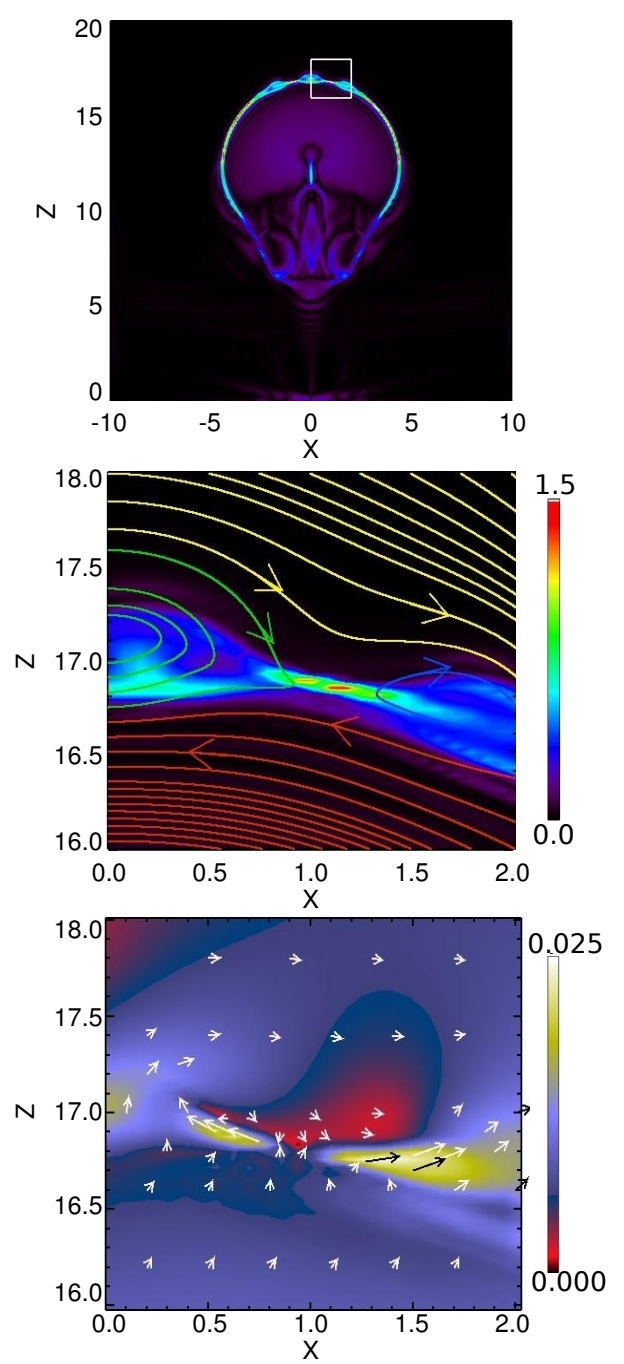

Figure 15. Current density and plasma velocity distribution at the $y=0$ cross-section of the twisted loop $\left(D^{*}\right)$ just after the onset of kink-instability. Top panel shows distribution of the current density $|j|$ in the $y=0$ plane crossing the twisted loop, while the middle panels shows selected magnetic field lines and $|j|$ distribution (colour scale) in and around one reconnection region (shown as white square at the top panel). Lower panel shows the in-plane velocity distribution (colour scale $-\sqrt{v_{\mathrm{x}}^{2}+v_{\mathrm{z}}^{2}}$ ). 
Having described the main features of the field and energy evolution in loops A-D, we now return to consideration of the nature of the energy dissipation process. This exhibits all features characteristic of magnetic reconnection, such as change of field line connectivity, localised dissipation sites and rapid conversion of magnetic energy into kinetic and internal energy. The change in field line connectivity can be clearly seen in Figures 13-14, and has been analysed for cylindrical models by Hood et al. (2009) and Gordovskyy and Browning (2011) who demonstrate reconnection of individual field lines. Indeed, in order to untwist a field line, the footpoint connectivity must change substantially. Furthermore, the plasma is substantially non-ideal only in a small fraction of the domain volume, hence the dissipation is localised (characteristic of reconnection rather than global Ohmic dissipation). This is demonstrated in Figure 8, which shows the percentage of the grid assigned anomalous resistivity (as a consequence of exceeding the critical current). Comparing with Figure 4, it may be seen that the anomalous resistivity fraction peaks in the same time intervals when the magnetic energy substantially decreases; in these same periods, kinetic energy also peaks, indicative of reconnection outflows.

Figure 15 shows the current and velocity distribution at the central crosssection of the twisted curved loop during the early nonlinear phase of the kink instability. It can be seen that there is a 'shield-like' layer of strong current formed above the top of the loop. This layer is not uniform, and contains several intense current sheets similar to those seen in 2D models with plasmoid reconnection (e.g. Loureiro et al., 2007). More detailed analysis of the magnetic and velocity fields within one such current sheet-like feature shows reversal of one component of the magnetic field with an X-point, and inflow-outflow velocity patterns characteristic of reconnection (Figure 15 middle and lower panels) - although it should be noted that this is a section of a $3 \mathrm{D}$ structure, and reconnection in 3D may differ from 2D models. Apparently, these current sheets are locations where the reconnection occurs. Since this current layer separates twisted and non-twisted field, magnetic reconnection in this layer is likely to be responsible for the radial expansion of kink-unstable twisted loops found earlier (Gordovskyy and Browning, 2011; Bareford et al., 2013; Gordovskyy et al., 2014), as the twisted field lines reconnect with ambient untwisted field. Furthermore, in both straight and curved loop models, there are also a fragmented current structure within the loops, which also consists of many reconnecting current sheets, as demonstrate by Hood et al. (2009).

\subsection{Stratified Atmosphere}

The loops mentioned so far all have a density and temperature that are initially uniform, where $n=1.2 \times 10^{15} \mathrm{~m}^{-3}$ and $T=4 \times 10^{3} \mathrm{~K}$. In order to investigate the effect of a stratified atmosphere, we also have loop $\mathrm{D}^{*}$, identical to loop $\mathrm{D}$, except that it has a non-uniform atmosphere based on the work by Gordovskyy et al. (2013), with density defined as

$$
\rho(z)=\rho_{1} \exp \left(\frac{-\left(z_{\mathrm{sh}}+z\right)}{z_{1}}\right)+\rho_{2} \exp \left(\frac{-\left(z_{\mathrm{sh}}+z\right)}{z_{2}}\right),
$$




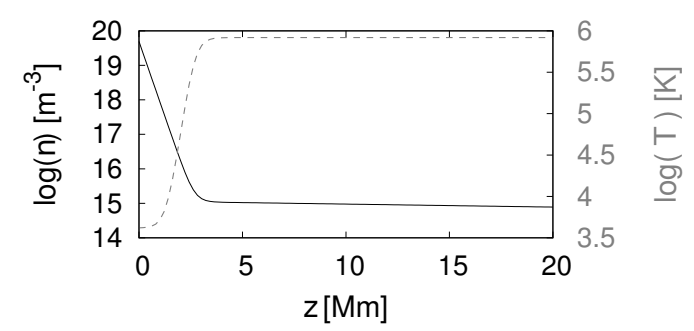

Figure 16. The initial density (black) and temperature (grey) with height $(z)$ for loop $D^{*}$.
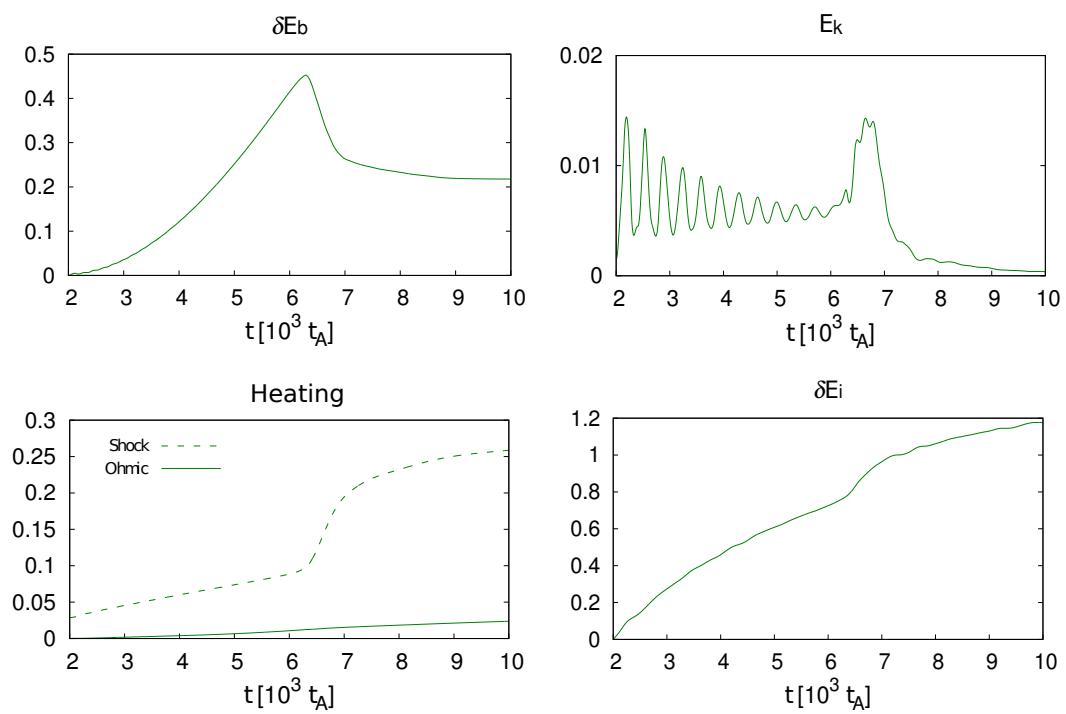

Figure 17. The change in magnetic energy (top left), the kinetic energy (top right), the cumulative Ohmic and shock heating (bottom left), and the change in internal energy (bottom right) for loop D*gravitation. Energies are volume intergrated.

where $\rho_{1}=3.34 \times 10^{-5} \mathrm{~kg} \mathrm{~m}^{-3}$ is the photospheric density, $\rho_{2}=2 \times 10^{-12} \mathrm{~kg} \mathrm{~m}^{-3}$ is the mean density in the corona, $z_{1}=0.25 \mathrm{Mm}$ is the density scale height between the photosphere and the transition region, $z_{2}=50 \mathrm{Mm}$ is the coronal scale height, and $z_{\mathrm{sh}}=1.5 \mathrm{Mm}$ simply allows the density profile to be shifted horizontally.

The initial atmosphere is shown in Figure 16 - the temperature profile follows from hydrostatic balance, and so a gravitational term $(\rho g(z))$ is added to the force Equation (4). Between $z=0$ and $z=4$ the temperature increases by more than two orders of magnitude (from around $4000 \mathrm{~K}$ to $0.83 \mathrm{MK}$ ), while the particle number density drops from $10^{20}$ to $10^{15} \mathrm{~m}^{-3}$. This region represents the chromosphere and transition region. The rest of the domain $(z>4)$ represents the corona, where the temperature and density are more or less constant. It is appropriate to add an atmosphere to loop D, since the increase in thermal pressure towards the footpoints is matched by the hundredfold increase in magnetic pressure. 
Increasing the density reduces the Alfvén speed, which has the unwelcome consequence of violating the constraint for direct current heating. At the footpoints, the driving speed (Figure 2, left) is now twice the Alfvén speed, and so the perturbations generated by the driving occur too frequently for the loop to have time to settle into an equilibrium. This issue is easily rectified if the driving factor, $\omega_{0}$, is reduced by an order of magnitude. Hence, $v_{\mathrm{A}}(z=0)$ is now five times the driving speed, which compares well with observed values for active region field strengths $(1 \mathrm{kG})$, photospheric flow speeds $\left(1 \mathrm{~km} \mathrm{~s}^{-1}\right)$ and densities $\left(4 \times 10^{-4} \mathrm{~kg} \mathrm{~m}^{-3}\right)$.

The stratified atmosphere used for loop $\mathrm{D}^{*}$ is not in equilibrium in the presence of thermal conduction; however, the atmosphere "settles down" (i.e., the heatflux due to conduction and, therefore, temperature changes become very small), if the model is left to evolve without driving for $2000 t_{\mathrm{A}}$. Only then do we begin the driving phase with $t_{\mathrm{tw}}=4300 t_{\mathrm{A}}$ set such that the driving ramps down at $6300 t_{\mathrm{A}}$. Note, no restart is required after the driving phase, since in order for the atmosphere to acquire its initial equilibirum, the simulation must have resistive MHD and conduction switched on from the start.

Although, in the present study we include only one model with atmospheric stratification, several more models are described in previous and forthcoming papers (Gordovskyy et al., 2014, 2015; Pinto et al., 2015), which focus on the velocity field, and thermal and non-thermal emission during magnetic reconnection in twisted loops in stratified atmosphere. Furthermore, there are several studies (Hood et al., 2012; Archontis et al., 2014, and references therein) considering evolution of twisted magnetic ropes in a stratified corona after emergence from the subphotospheric layers. Therefore, here we focus only on the effect of stratification on loop stability.

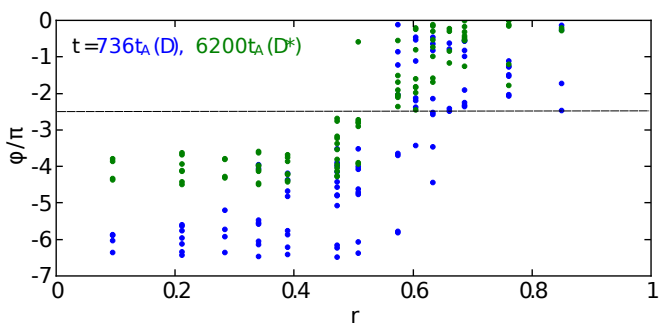

Figure 18. The magnetic twist in units of $\pi$ as a function of radial distance from footpoint centre for loops $\mathrm{D}$ (blue) and $\mathrm{D}^{*}$ (green). The format of this figure follows that used for Figure 3.

The energy plots begin from when the twisting, leading to creation of kinkunstable loop, is started. Figure 17 (top left) shows that the driving takes $4300 t_{\mathrm{A}}$ to induce an instability. Comparison with loop D (Figure 10), reveals that the presence of an atmosphere requires less buildup in magnetic energy for instability onset, and so the resulting energy release is half that seen for loop D. This also means that adding a stratified atmosphere reduces the twist required for the instability onset: just before the kink instability loop $\mathrm{D}^{*}$ has a lower twist for $r \leq 0.5$ compared to loop $\mathrm{D}$. The kinetic energy plot for loop $\mathrm{D}^{*}$ exhibits the 
expected peak at the time the instability occurs, but there is also a wave pattern that, during the driving phase, decays in amplitude. Figure 17 (bottom left) shows that shock heating continues to dominate over Ohmic heating. Internal energy undergoes a continual increase, the gradient of which steepens as the loop goes unstable. The heating before the instability is possibly connected to the decaying wave form seen in the kinetic energy plot. Although disguised by the resolution of the time axis, the relaxation phase is even more extended than it is for loop D. The relaxation time is roughly $1000 t_{\mathrm{A}}$, which equates to a dimensionalised time of $355 \mathrm{~s}$ if one uses the scalings given in Section 3.

\section{Summary and Conclusions}

We have investigated the magnetic reconnection and energy release in twisted magnetic fluxtubes representing coronal loops. The twist has been created by rotating footpoints of initially potential fluxtubes. The developed models allow us to study the effect of magnetic configuration (magnetic field convergence near footpoints and its large-scale curvature) and atmospheric stratification on fluxtube stability, and energy release. In the present paper we have used five different fluxtube models with configurations similar to those developed in (Browning et al., 2008; Hood et al., 2009; Gordovskyy and Browning, 2011; Bareford et al., 2013; Gordovskyy et al., 2014). Other models with different parameters have been considered recently by Gordovskyy et al. (2015) and Pinto et al. (2015).

Based on the present numerical experiments, we can make several conclusions.

Firstly, the kink instability occurs in all five experiments. Although the magnetic energy decrease is noticeably different in different experiments, the evolution of magnetic field when the energy starts to drop is consistent with the kink instability. Secondly, all considered models demonstrate change of magnetic connectivity along with conversion of magnetic energy into kinetic and internal energy, while the resistivity is substantially non-zero only in a small fractions of domain volumes. Hence, the kink instability results in magnetic reconnection in all cases.

Further, we can conclude that, broadly, the predictions of earlier idealised models of non-linear kink instability (in cylindrical fluxtubes) are valiad for more realistic configurations. At the same time, there are some factors resulting in noticeable differences, as described below.

The large-scale curvature affects the geometry of magnetic reconnection. Similar to the straight fluxtubes, in curved loops the kink instability results in a formation of helically-shaped structure with high current density. However, the current distribution in these models loses its cylindrical symmetry, with strong currents formed in a thin shield above the loop top. Additionally, there are regions with very high current density above the footpoints in the loops with strong convergence (models D and $\mathrm{D}^{*}$ ). The spatial distribution of heated plasma usually follows the distribution of strong currents. Hence, in the straight fluxtubes A and B, heated plasma is rather uniformly distributed along the fluxtubes. At the same time, in curved loops plasma heating is more localised: there is a strong heating near the loop top in model $\mathrm{C}$, while strongly converging loops $\mathrm{D}$ 
and $\mathrm{D}^{*}$ show very high temperatures near the footpoints. The localised heating is, of course, mitigated by thermal conduction, which smoothes the temperature distribution along field lines, while it remains very structured across field lines.

The twist angle just before the kink instability (the critical angle) can be used as a measure of fluxtube stability. The critical angle in curved fluxtubes (models $\mathrm{C}, \mathrm{D}$ and $\mathrm{D}^{*}$ ) is systematically lower than in straight fluxtubes (models A and B). Hence, the large-scale curvature reduces the stability of fluxtubes. This is most likely due to the additional field line curvature in bended loops. Additionally, the absence of cylindrical symmetry can have a destabilising influence.

The effect of footpoint convergence on fluxtube stability is more ambiguous, as there are several factors involved. Fluxtubes with larger average radius are more stable, which means that fluxtubes with converging field should be more stable. This has been previously shown analytically by Browning and Van der Linden (2003) and Bareford et al. (2011). On the other hand, fluxtubes with expanding field (i.e. converging near footpoints) and the same magnetic flux can have higher magnetic energy density in the azimuthal field component, which makes them less stable. The results for curved and straight fluxtubes demonstrate different behaviours. Thus, loop D with strongly converging footpoints is more stable than loop $\mathrm{C}$ with weak convergence. At the same time, non-converging fluxtube $\mathrm{A}$ appears to be slightly more stable than weakly converging fluxtube B. Therefore, more experiments are needed to study the effect of footpoint convergence.

The inclusion of atmospheric stratification is found to reduce the stability of twisted loops. The main reason for this is that fast changes in the strongly inhomogeneous medium give rise to waves propagating from the dense chromosphere into the corona, which have a destabilising effect.

Finally, an important methodological implication from our study is that the shock viscosity needs to be taken into account as an additional dissipation chanel. Although, the shock viscosity in LARE3D (and in many other) MHD simulations does not represent the real viscosity in the solar corona, it appears to represent additional Ohmic dissipations in slow magnetoacoustic shocks, which is a real physical effect. This issue is particularly important for numerical experiments with localised resistivity effects, as shocks would alter the effective spatial resistivity distribution.

Acknowledgements This work is funded by Science and Technology Facilities Council (UK). The simulations were run on the UK MHD Consortium cluster based in St Andrews and on the COSMA Data Centric system at Durham University. The latter is operated by the Institute for Computational Cosmology on behalf of the STFC DiRAC HPC Facility (www.dirac.ac.uk). This equipment was funded by a BIS National E-infrastructure capital grant ST/K00042X/1, DiRAC Operations grant ST/K003267/1 and Durham University. DiRAC is part of the National E-Infrastructure.

\section{References}

Arber, T.G., Longbottom, A.W., Gerrard, C.L., Milne, A.M.: 2001, J. Comp. Phys. 171, 151. 
Archontis, V., Hood, A.W., Tsinganos, K.: 2014, Astrophys. J. 778, 42.

Bareford, M.R., Browning, P.K., Van der Linden, R.A.M.: 2010, Astron. Astrophys. 521, 70.

Bareford, M.R., Browning, P.K., Van der Linden, R.A.M.: 2011, Solar Phys. 273, 93.

Bareford, M.R., Hood, A.W., Browning, P.K.: 2013, Astron. Astrophys. 550, 40.

Bareford, M.R., Hood, A.W.: 2015, Phil. Trans. Roy. Soc. A. 373, 20140266.

Botha, G.J.J., Arber, T.D., Hood, A.W.: 2011, Astron. Astrophys. 525, A96.

Braginskii, S.I.: 1965, Rev. Plasma Phys. 1, 205.

Brio, M., Wu, C.C.: 1998, J. Comp. Phys. 75, 400.

Brosius, J.W., Dow, A.N., Rabin, D.M.: 2014, Astrophys. J. 790, 112.

Browning, P.K., van der Linden, R.A.M.: 2003, Astron. Astrophys. 400, 355

Browning, P.K., Gerrard, C., Hood, A.W., Kevis, R., van der Linden, R.A.M.: 2008, Astron. Astrophys. 485, 837.

Cargill, P.J.: 2013, Nature 493, 485.

Cirtain, J.W., Golub, L., Winebarger, A.R., de Pontieu, B., Kobayashi, K., Moore, R.L. et al.: 2013, Nature 493, 501.

Gordovskyy, M., Browning, P.K.: 2011, Astrophys. J. 729, 101.

Gordovskyy, M., Browning, P.K.: 2012, Solar Phys. 277, 299.

Gordovskyy, M., Browning, P.K., Kontar, E.P., Bian, N.H.: 2013, Solar Phys. 284, 489.

Gordovskyy, M., Browning, P.K., Kontar, E.P., Bian, N.H.: 2014, Astron. Astrophys. 561, 72.

Gordovskyy, M., Kontar, E.P, Browning, P.K.: 2015, Astron. Astrophys., submitted.

Hood, A.W., Priest, E.R.: 1979, Solar Phys. 64, 303.

Hood, A.W.: 1992, Plasma Phys. Control. Fusion 34, 411.

Hood, A.W., Browning, P.K., Van der Linden, R.A.M.: 2009, Astron. Astrophys. 506, 913.

Hood, A.W., Archontis, V., MacTaggart, D.: 2012, Solar Phys. 278, 3.

Klimchuk, J.A.: 2000, Solar.Phys. 193, 53.

Kumar, P., Cho, K.S.: 2014, Astron. Astrophys. 572, A83.

Kuridze, D., Mathioudakis, M., Kowalski, A.F., Keys, P.H., Jess, D.B., Balasubramaniam, K.S., Keenan, F.P.: 2013, Astron. Astrophys. 552, A55.

Loureiro, N.F., Schekochihin, A.A., Cowley, S.C.: 2007, Phys. Plasmas 14, 100703.

Mellor, C, Gerrard, C.L., Galsgaard, K., Hood, A.W., Priest, E.R.: 2005, Solar Phys. 227, 39. Parker, E.N.: 1988, Astrophys. J. 330, 474.

Parnell, C.E., De Moortel, I.: 2012, Phil. Trans. Roy. Soc. A 370, 3217

Peter, H., Bingert, S.: 2012, Astron. Astrophys. 548, 1.

Pinto, R., Gordovskyy, M., Browning, P.K., Vilmer, N.: 2015, Astron. Astrophys. submitted.

Reale, F.: 2014, Living Rev. Solar Phys. 11 http://solarphysics.livingreviews.org/Articles/ Irsp-2014-4/

Srivastava, A.K., Zaqarashvili, T.V., Kumar, P., Khodachenko, M.L.: 2010, Astrophys. J. 715, 292.

Van Leer, B.: 1997, J. Comput. Phys. 135, 229.

Wang, H., Cao, W., Liu, C., Xu, Y., Liu, R., Zeng, Z., Chae, J., Ji, H.: 2015, Nature Comm. $\mathbf{7}, 7008$.

Wilkins, M.L.: 1980, J. Comput. Phys. 36, 281.

Yan, X.L., Xue, Z.K., Liu, J.H., Kong, D.F., Xu, C.L.: 2014, Atrophys. J. 797, 52. 